\title{
Research on the Application Observation of Standardized Full-course Nursing Intervention in the Perioperative Period of Minimally Invasive Arthrocentesis Surgery in Patients with Breast Fibroma
}

\author{
Mei Wang, YangYang, Shiqing Sun ${ }^{1}$, Fangcao He, Congcong Li \\ Department of Galactophore, Henan Province Hospital of Traditional Chinese Medicine, Zhengzhou 450002, \\ China
}

Keywords: Breast Fibroma; Minimally Invasive Atherectomy by Isolamer; Standardized Full-course Nursing Intervention

\begin{abstract}
Objective: To explore the effect of standardized nursing interventions in the perioperative period of minimally invasive arthroscopy of areola fibroids in patients with breast fibroids. Methods: 94 patients with breast fibroma were selected in our hospital from August 2015 to February 2018. According to the order of admission, there were 47 cases. All cases were treated with minimally invasive aortic ring resection of isolame, and the control group was given routine nursing during the perioperative period. The experimental group was given a standardized full-course nursing intervention. The incidence of postoperative complications, satisfaction with nursing work, and quality of life before and after intervention was compared between the two groups. Results: The incidence rate of postoperative complications in the experimental group was $6.38 \%$ (3/47) lower than that in the control group (25.53\%, 12/47). The satisfaction rate of nursing work was $97.87 \%$ (46/47) higher than that of the control group $(80.85 \%, 38 / 47)$. $(\mathrm{P}<0.05)$; There was no significant difference in the quality of life scores between the two groups before intervention $(\mathrm{P}>0.05)$, and the quality of life scores in the experimental group were higher than those in the control group $(\mathrm{P}<0.05)$. Conclusions: Standardized breastfeeding therapy for breast fibroma patients undergoing minimally invasive amelioration of the areola with Maimaton can significantly reduce the incidence of postoperative complications, effectively improve the quality of life of patients, and have high satisfaction with nursing work. It is worthy of clinical application. .
\end{abstract}

\section{Introduction}

Breast fibroma is a common clinical benign breast lesion, which occurs in breast lobule and mammary gland epithelium. It can be divided into ordinary fibroadenoma, pure fibroadenoma and giant fibroadenomas. It is more common in women of childbearing age, and is healthy and lives for women. Quality has a serious impact [1]. At present, surgical resection is the first choice for the clinical treatment of breast fibroids, and the minimally invasive aortic ring through the areola is the most commonly used, but the operation has certain risks, and postoperative wound infection, liquefaction and other complications have a high incidence, affecting patients Prognosis [2]. Some studies have pointed out that the corresponding nursing measures for breast fibroid surgery patients can help postoperative complications, such as incision infection, hematoma infection, etc., and promote postoperative rehabilitation [3]. In recent years, due to a variety of factors, the incidence of breast fibroids has continued to climb. In order to ensure the effectiveness of the surgery and accelerate postoperative recovery, perioperative nursing interventions are highly concerned by medical personnel. Therefore, the selection of an effective, reasonable, and feasible nursing model is particularly critical for patients with breast fibroids undergoing minimally invasive atropotomy. In this study, 94 patients with breast fibroma were selected to analyze the effect of standardized full-term nursing intervention in patients with breast fibroids during the perioperative period of minimally invasive arthroscopy with areola. The result is as follows. 


\section{Materials and Methods}

\subsection{Clinical Data.}

Select 94 cases of breast fibromatosis in our hospital from August 2015 to February 2018, according to the order of admission, 47 cases each. The experimental group was aged 23 to 37 years, mean age (30.26 \pm 3.12$)$ years, course of disease 5 months to 5 years, mean duration of disease (2.84 \pm 1.25$)$ years, and control group age of 24 to 38 years, mean age (20.89 \pm 3.45$)$. At the age of 6 months to 5 years, the average course of disease was $(2.92 \pm 1.36)$ years. This study was reviewed and approved by the Institute of Ethical Society of our institute, and there was no significant difference in baseline data (age, course) between the two groups $(\mathrm{P}>0.05)$.

\subsection{Methods.}

They were all treated with the isolamomycoma minimally invasive atherectomy. The control group was given routine perioperative care, such as assisting the patient in various preoperative examinations, instructing the patient to select an appropriate position for surgery, and instructing him to cooperate with surgery and anesthesia-related issues. The surgeon actively cooperated with the surgeon to complete the operation and performed well after the operation. Incision care, to guide patients to perform daily activities, etc.; experimental group to be standardized full-care intervention, including (1) preoperative care: 1 operative time, accurate grasp of the patient's menstrual cycle, select the operation 4 to 5 days after menstruation; 2 psychological care, Due to lack of awareness of breast fibroids and minimally invasive aorticotomy by breast cancer, there are many negative emotions such as tension and anxiety. Nursing personnel need to give patients detailed information about diseases and surgery, and guide them to raise doubts. And to be targeted answers, if necessary, to invite successful cases of similar diseases to share the experience of rehabilitation, enhance the patient's treatment confidence, make it actively face treatment, to ensure the smooth operation; 3 preoperative preparation to ensure that the operating room environment is suitable, nursing staff guide patients to do Good preoperative preparation guides patients to drink plenty of water before surgery to maintain metabolism; Exploring the condition of chest lumps, and preparing drainage for those with larger lumps; (2) Intraoperative care: The nursing staff closely monitors the patient's vital signs and cooperates with the surgeon. If an emergency occurs, keep the sedation and cooperation with the surgeon; The mass is larger and needs drainage, try to drain as often as possible; (3) postoperative care: 1 basic care, closely monitor the patient's vital signs such as blood pressure, respiration, and pulse, wait for the patient to wake up and change to regular observation; pay strict attention to the incision , Help them to complete dressing, turn over, etc. may pull wounds and provide good life care; to provide patients with high-vitamin, high protein, high calorie and other nutritious foods, fasting spicy greasy food, promote wound healing; 2 incision care, regular inspection Incision bandages to prevent over-tightening or loosening; care to keep accessories dry and clean during the care process to avoid infection; after 24 to $48 \mathrm{~h}$ pull out the drainage tube and do compressive hemostasis; 3 health guidance, before discharge, nursing staff Inform patients of post-discharge precautions and ensure that they are fully informed and encourage patients to perform activities as soon as possible; help patients understand surgery correctly And postoperative conditions may occur to avoid negative emotions and affect postoperative recovery. Follow-ups were conducted regularly to guide patients to the hospital.

\subsection{Observation Indicators.}

(1) Postoperative complications, including incision infection, incision liquefaction, and hematoma infection. (2) Quality of life: The SF-36 measures the quality of life from the aspects of physiological function, social function, mental health, and emotional function. [4]. (3) Satisfaction of nursing work was assessed by self-made nursing job satisfaction scale with a total score of 100 points, which was divided into satisfaction (>90 points), general satisfaction (80 to 90 points), and dissatisfaction $(<80$ points $)$. Total satisfaction $=($ satisfaction + general satisfaction $) /$ total number of cases $\times 100 \%$. 


\subsection{Statistical Methods.}

SPSS17.0 analysis, measurement data with ( \pm s) said, t test, count data with $\mathrm{n}(\%)$ said, chi-square test, test standard $\alpha=0.05$.

\section{Results}

\subsection{Postoperative Complications.}

Comparing the incidence of postoperative complications in the two groups, the $6.38 \%$ in the experimental group was lower than the $25.53 \%$ in the control group $(\mathrm{P}<0.05)$. See Table 1.

Table 1 Comparison of postoperative complications between the two groups [n(\%)]

\begin{tabular}{|c|c|c|c|c|c|c|}
\hline Groups & $\begin{array}{l}\text { Number } \\
\text { cases }\end{array}$ & of & Liquefaction & $\begin{array}{l}\text { Incision } \\
\text { infection }\end{array}$ & $\begin{array}{l}\text { Hematoma } \\
\text { infection }\end{array}$ & $\begin{array}{l}\text { Total } \\
\text { incidence }\end{array}$ \\
\hline test group & 47 & & $1(2.13)$ & $2(4.26)$ & $0(0.00)$ & $3(6.38)$ \\
\hline $\begin{array}{l}\text { Control } \\
\text { group }\end{array}$ & 47 & & $4(8.51)$ & $5(10.64)$ & $3(6.38)$ & $12(25.53)$ \\
\hline & $\square$ & & $\square$ & $\square$ & $\square$ & $\begin{array}{l}6.425 \\
0.011\end{array}$ \\
\hline
\end{tabular}

\subsection{Quality of Life.}

There was no significant difference in quality of life scores between the two groups before the intervention $(\mathrm{P}>0.05)$. The scores of quality of life after intervention in the experimental group were higher than those in the control group $(\mathrm{P}<0.05)$. See Table 2.

Table 2 Comparison of scores for each dimension of quality of life in the two groups ( \pm s, points)

\begin{tabular}{|c|c|c|c|c|c|c|}
\hline Time & Groups & $\begin{array}{l}\text { Number of } \\
\text { cases }\end{array}$ & $\begin{array}{l}\text { Physiological } \\
\text { function }\end{array}$ & Mental Health & Social function & $\begin{array}{l}\text { Emotional } \\
\text { function }\end{array}$ \\
\hline \multirow{4}{*}{$\begin{array}{l}\text { Before } \\
\text { intervention }\end{array}$} & test group & 47 & $50.14 \pm 5.26$ & $50.63 \pm 5.74$ & $51.32 \pm 6.74$ & $50.47 \pm 5.80$ \\
\hline & Control & 47 & $50.89 \pm 5.37$ & $50.38 \pm 5.62$ & $50.92 \pm 6.41$ & $51.12 \pm 6.04$ \\
\hline & $t$ & - & 0.684 & 0.213 & 0.295 & 0.532 \\
\hline & $P$ & - & 0.496 & 0.832 & 0.769 & 0.596 \\
\hline \multirow{4}{*}{$\begin{array}{l}\text { After } \\
\text { intervention }\end{array}$} & test group & 47 & $75.21 \pm 6.30$ & $74.29 \pm 6.32$ & $75.60 \pm 5.98$ & $76.19 \pm 6.30$ \\
\hline & $\begin{array}{l}\text { Control } \\
\text { group }\end{array}$ & 47 & $69.87 \pm 5.90$ & $68.14 \pm 6.47$ & $70.14 \pm 6.02$ & $70.28 \pm 6.19$ \\
\hline & $t$ & - & 4.242 & 4.662 & 4.411 & 4.588 \\
\hline & $P$ & - & 0.000 & 0.000 & 0.000 & 0.000 \\
\hline
\end{tabular}

\subsection{Nursing Job Satisfaction.}

In the experimental group, the satisfaction rate of the nursing work was $97.87 \%$ higher than that of the control group $(80.85 \%)(\mathrm{P}<0.05)$. See Table 3 .

Table 3 Comparison of satisfaction between two groups of nursing work [n(\%)]

\begin{tabular}{|c|c|c|c|c|c|}
\hline Groups & $\begin{array}{l}\text { Number of } \\
\text { cases }\end{array}$ & satisfaction & $\begin{array}{l}\text { Generally } \\
\text { satisfied }\end{array}$ & Not satisfied & $\begin{array}{l}\text { Total } \\
\text { satisfaction }\end{array}$ \\
\hline test group & 47 & $35(74.47)$ & $11(23.40)$ & $1(2.13)$ & $46(97.87)$ \\
\hline $\begin{array}{l}\text { Control } \\
\text { group }\end{array}$ & 47 & $24(51.06)$ & 14(29.79) & $9(19.15)$ & $38(80.85)$ \\
\hline$\chi^{2}$ & 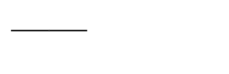 & 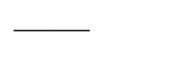 & 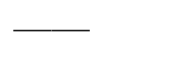 & $\square$ & $\begin{array}{l}7.162 \\
\end{array}$ \\
\hline
\end{tabular}




\section{Discussion}

Breast fibroma is a common gynecological disease, multiple diseases, the main early stage of the performance of breast lumps, but the tumor is small, there is no pain, many patients are difficult to detect, when the tumor gradually increases, the patient's breast pain is severe, affecting daily life [5]. At this stage, the clinical use of surgical removal of breast fibroids, including minimally invasive atraucutomy by the areola Mammoth can completely remove breast tumors, the effect is more significant, has become the preferred treatment of breast fibroids [6]. However, studies have shown that although surgical resection is simple and easy to perform, if it is not supplemented with relevant nursing measures, it will also have a certain impact on the prognosis of patients [7]. In addition, the treatment of breast fibroids by the isolatoid Mammoto minimally invasive avulsion surgery lacks good and good nursing intervention, which can easily lead to postoperative complications and seriously affect the physical and mental health and quality of life of patients.

In this study, standardized breastfeeding patients with breast fibroids undergoing minimal-invasive aspiration of the areola were treated with standardized nursing interventions. The results were satisfactory. Standardized whole-course nursing intervention is a kind of nursing intervention mode that takes quality nursing concept as the working principle, taking into account the physiological and psychological state of the patient, and emphasizing systematic nursing work, providing comprehensive nursing services for patients, and making nursing measures more pertinent [8]. In addition, the standardized whole-course nursing intervention believes that the general surgery patient care should be started by determining the surgical plan, and then provide perioperative nursing interventions, and provide targeted guidance on the patient's postoperative life habits, such as rest instructions, diet plans, Regular review and so on. The results of this study showed that the incidence rate of postoperative complications in the experimental group was 6.38\% lower than that of the control group, which fully demonstrated that the standardized full-course nursing intervention can effectively reduce postoperative concomitant breast fibroma patients undergoing minimally invasive aproplastomy. Analysis of the reasons may be due to the standardization of the whole process of nursing interventions, the nurses informed patients before surgery, precautions, possible complications and their response measures, postoperative incision care, prevention of incision infection and incision liquefaction and other complications, And closely monitor the patient's vital signs, when abnormalities occur, maintain sedation, and take timely symptomatic treatment, can effectively reduce postoperative complications. In addition, the scores of quality of life and the degree of satisfaction with nursing work after intervention in the experimental group were higher than those in the control group, suggesting that standardization of nursing intervention for breast fibroid patients undergoing minimally invasive atraucutomy of breast cancer can significantly improve the lives of patients. Quality, improve nursing job satisfaction. The reason is that standardized general nursing interventions follow general surgery breast fibroids, and nursing care interventions are carried out through the iscolysmic minimally invasive auricular atherectomy interventions. At the same time, symptomatic care measures are provided based on the patient's condition and actual needs, so as to promote postoperative rehabilitation. Improve the quality of life and further improve the satisfaction of nursing work.

In summary, standardized breastfeeding intervention for breast fibroma patients undergoing minimally invasive atraucutomy of areola breasts can significantly reduce the occurrence of postoperative complications, effectively improve the quality of life of patients, and have higher satisfaction with nursing work.

\section{Acknowledgements}

Fund Project: Study on the efficacy and mechanism of Shenyan Pill combined with tamoxifen in inhibiting the growth of breast tumor and preventing lung metastasis based on traditional Chinese medicine "Consumption method", 13A360610 


\section{References}

[1] Wang Honghui, Wang Yujie, Shan Xinfang, et al. Observation of curative effect of breast fibroids assisted by single-endoscopic resection of anterior mastectomy through breast hernia [J].Chinese Journal of Clinical Practical Medicine, 2015, 6(4):16-18.

[2] Jiang Xiaohong,Zhao Xia,Huang Weiying.Evaluation of clinical nursing intervention in patients with breast fibroid surgery[J].China Practical Medicine,2016,11(10):251-252.

[3] Wang Fang. Clinical nursing intervention in breast fibroid surgery [J]. Chinese modern doctors, 2015, 53 (4): 151.

[4] Ren Chunwu, Chen Liming. Effects of individualized health education on blood pressure fluctuations and SF-36 scale scores in elderly hypertensive patients [J]. Chinese Medical Innovation, 2015, 12(19):108-110.

[5] Li Han. Observation of the efficacy of mammotoma-guided minimally invasive mammoplasty in the treatment of breast fibroadenoma patients with molybdenum target positioning [J]. Chinese Journal of Chinese Medical Science, 2016, 28(4):38-39.

[6] Mo Haiyang. Comparison of clinical efficacy of benign breast mass treated by areola incision and minimally invasive rotation system with traditional surgery [J]. Henan Journal of Surgery, 2017, 23(4):86-87.

[7] Liu Jing. Clinical nursing intervention observation in breast fibroid surgery [J]. Contemporary medicine, 2017, 23 (12): 151-152.

[8] Ye Peiping, Wu Jiangqiong, Luo Shuzhen. Effects of standardized nursing interventions for parturient women on outcomes of delivery and cognitive performance during labor [J]. Journal of Mathematical Medicine, 2015, 28(7):1058-1059. 